\title{
Comparación de dos Versiones Reducidas de la Escala PANAS: Análisis Factoriales en una Muestra Argentina
}

\author{
Comparison of two Short-Forms of the PANAS: Factor Analysis in a Argentine \\ Sample
}

\author{
Pablo Ezequiel Flores-Kanter ${ }^{1}$ y Leonardo Adrián Medrano ${ }^{1}$
}

\begin{abstract}
Resumen
El afecto y su implicancia en la cognición constituye un área de investigación que se ha desarrollado mucho el último tiempo (Davidson, Scherer, \& Goldsmith, 2009). Dada su importancia, se vislumbra la necesidad de contar con instrumentos confiables y válidos que permitan medir el afecto (Gray \& Watson, 2007). En este sentido, unos de los instrumentos más mencionados en la literatura científica es la Escala de Afecto Positivo y Negativo (PANAS; Watson, Clark, \& Tellegen, 1988). Recientemente se han propuesto dos versiones reducidas del PANAS, una versión Inglesa (Thompson, 2007) y otra Portuguesa (Galinha, Pereira, \& Esteves, 2014). El presente trabajo propone evaluar y comparar estas versiones reducidas del PANAS, con el objetivo de verificar cuál de éstas se adapta mejor al contexto Argentino. Los resultados obtenidos avalan la utilización de la Versión Reducida del PANAS, integrada por los ítems de la Versión Portuguesa.
\end{abstract}

Palabras clave: adaptación Argentina, análisis factorial confirmatorio, análisis factorial exploratorio, versión reducida del PANAS

\begin{abstract}
Affects and its implications in cognition is a research area that has a long developed the last time (Davidson, Scherer, \& Goldsmith, 2009). Given its importance, its seem the need for reliable and valid instruments to measure the affect (Gray, \& Watson, 2007). In this sense, the Positive and Negative Affect Schedule is one of the most instruments mentioned in the scientific literature (PANAS; Watson, Clark, \& Tellegen, 1988). Recently, they were proposed two short-forms of PANAS, an English version (Thompson, 2007) and one Portuguese versión (Galinha, Pereira, \& Esteves, 2014). The present study aims to evaluate and compare these short-forms, in order to verify which of these is better adapted to the Argentine context. The results support the use of the short version of the PANAS, composed with items of the Portuguese version.
\end{abstract}

Keywords: Argentine adaptation, confirmatory factor analysis, exploratory factor analysis, PANAS shortform

\footnotetext{
${ }^{1}$ Secretaría de Investigación, Universidad Siglo 21, Argentina. Correo: ezequielfk@ gmail.com

Revista Iberoamericana de Diagnóstico y Evaluación - e Avaliação Psicológica. RIDEP · N49 · Vol.4 · 37-46 · 2018

ISSN: $1135-3848$ print /2183-6051online
} 


\section{Introducción}

La experiencia afectiva o el afecto es una parte importante y prevaleciente en la vida diaria de las personas (Gargurevich, 2010; Watson, 2000). El afecto comprende una categoría general que incluye tanto a las emociones como a los estados anímicos (Bower \& Forgas, 2003; Hervás \& Vázquez, 2006). Asimismo, muchas veces la palabra afecto se usa como sinónimo de sentimiento, esto es, asociándola a la experiencia afectiva; característica compartida a su vez por los estados de ánimo como por las emociones (Watson, 2000). Puede decirse entonces que cuando se habla del afecto se está haciendo referencia de manera general a un tipo de experiencia afectiva, a un sentimiento, que puede tener que ver con emociones o estados de ánimo, y que puede ser tanto positivo como negativo (Hervás \& Vázquez, 2006; Moreira \& Gamboa, 2016; Watson, 2000).

El afecto y su implicancia en la cognición constituye un área de investigación que se ha desarrollado mucho el último tiempo (Davidson, Scherer, \& Goldsmith, 2003; Eich, Kihlstrom, Bower, Forgas, \& Niedenthal, 2003). De este modo, las diversas investigaciones han dado a conocer la influencia que ejercen las emociones y los estados anímicos sobre la memoria (Bower \& Forgas, 2001; McGaugh \& Gahill, 2009; Reisberg \& Hertel, 2004), la elaboración de juicios (Flores Kanter, Medrano, \& Conn, 2015; Flores Kanter, Medrano, \& Manoiloff, 2014; Forgas, 2001; 2003; Medrano, Flores Kanter, Pereno, \& Moretti, 2015), y la toma de decisiones (Damasio, 2005; Loewenstein \& Lerner, 2003; Rolls, 2014). Dada su importancia, se vislumbra la necesidad de contar con instrumentos confiables y válidos que permitan medir el afecto (Gray \& Watson, 2007; Moreira \& Gamboa, 2016; Watson, 2000).

En relación con lo anterior, unos de los instrumentos más mencionados en la literatura científica es la Escala de Afecto Positivo y Negativo (PANAS; Watson, Clark, \& Tellegen, 1988). La escala PANAS consta de 20 ítems, 10 ítems que miden el Afecto Positivo (e.g Entusiasmado; Activo; Interesado) y 10 ítems que miden el Afecto Negativo (e.g. Irritable; Temeroso; Afligido). Esta escala parte de un modelo dimensional, donde se plantea que el afecto se compone de dos factores de orden superior: Afecto Positivo y Afecto Negativo. El modelo plantea además la unipolaridad e independencia de ambos factores (Gray \& Watson, 2007; Watson, 2000).

Aunque diversos estudios de orden exploratorio y confirmatorio han ubicado a la escala PANAS como uno de los instrumentos de mayor confianza y validez para medir el afecto positivo y negativo (Flores Kanter \& Medrano, 2016; Galinha, Pereira, \& Esteves, 2013; Gray \& Watson, 2007; Medrano, Flores Kanter, Trógolo, Curarello, \& Gonzales, 2015; Moriondo, De Palma, Medrano, \& Murillo, 2012; Terracciano, Mc Crae, \& Costa, 2003; Tuccitto, Giacobbi, \& Leite, 2010; Watson, 2000), se han propuesto otras versiones del PANAS. Entre éstas se encuentra la versión reducida (short-form), que se ha desarrollado con el objeto de facilitar las respuestas en cuestionarios muy extensos que envuelven muchas variables, o en contextos donde hay limitaciones de tiempo, como puede ser el organizacional o educacional (Galinha, et al. 2014). Esta versión reducida del PANAS ha sido presentada en diversos formatos, los cuales comparten el hecho de contener sólo 10 ítems (5 ítems referidos al Afecto Positivo y 5 ítems referidos al Afecto Negativo) pero difieren en cuanto a los estados afectivos de orden inferior que incluyen (Galinha, et al., 2014; Gargurevich, 2010; Gyollai, Simor, Köteles, \& Demetrovics, 2011; Merz, et al., 2013; Karim, Weisz, \& Rehman, 2011; Thompson, 2007).

Una de las versiones cortas del PANAS más citadas es la versión internacional propuesta por Thompson (2007). Haciendo uso de análisis cualitativos y cuantitativos este último autor propuso una escala compuesta por 10 adjetivos, 5 correspondientes al Afecto Positivo y 5 correspondientes al Afecto Negativo. Los adjetivos correspondientes al afecto positivo fueron: Alerta, Inspirado, Determinado, Atento, y Activo; mientras que los adjetivos referidos al afecto negativo fueron: Afligido, Hostil, Avergonzado, Nervioso, y Temeroso. El análisis factorial exploratorio de la escala, mediante componentes principales y rotación Varimax, dio cuenta de dos factores, el afecto positivo y negativo. Además, la escala presento coeficientes alfas Cronbach de .76 para el Afecto Negativo, y 
de .78 para el Afecto Positivo. Se llevo a cabo también un análisis factorial confirmatorio, en donde se evidenció que la estructura de dos factores mostraba buenos índices de ajustes ( $G F I=.94 ; A G F I=.90 ; C F I=.94 ; R M S E A=.066)$. El autor validó la escala en sucesivas muestras de habla Inglesa nativa y no nativa, encontrando resultados similares a los descritos. Además, esta versión ha sido validada en diferentes contextos, como ser Francia y Pakistan (Karim, et al. 2011), Perú (Gargurevich, 2010), Estados Unidos (Merz, et al., 2013) y Hungría (Gyollai, et al. 2011).

Recientemente Galinha, et al. (2014) han propuesto una versión Portuguesa reducida del PANAS. La misma consta también de 10 ítems, cinco de los cuales miden el afecto positivo, y los cinco restantes el afecto negativo. Con el objetivo de seleccionar los ítems que mejor representaban el afecto positivo y negativo en el léxico portugués, respetar la diversidad en las categorías emocionales representadas, y procurar una equivalencia con la versión inglesa de Thompson (2007), se incluyeron los siguientes ítems en la escala. Para el afecto positivo se consideraron los adjetivos Entusiasmado, Inspirado, Determinado, Activo e Interesado. Mientras que para el afecto negativo se incluyeron los siguientes ítems: Asustado, Temeroso, Afligido, Nervioso y Culpable. Mediante un análisis factorial confirmatorio los autores encontraron que el modelo bifactorial ajustaba adecuadamente a los datos $\left(X_{35}^{2}=45.1, \quad p=.119 ; \quad C F I=.99 ; \quad G F I=.96\right.$; $R M S E A=.04 ; S R M R=.05)$. La escala fue evaluada posteriormente en una muestra diferente encontrando resultados similares a los señalados (Galinha, et al. 2014).

En función de la importancia de contar con una versión corta del PANAS, el presente trabajo propone evaluar las dos versiones reducidas de este instrumento presentadas en los párrafos anteriores, con el objetivo de verificar cuál de éstas se adapta mejor al contexto Argentino. Si bien los estudios antecedentes presentan evidencia del adecuado ajuste de ambas escalas reducidas (i.e. versión Portuguesa e Inglesa), ninguno de éstos ha presentado una comparación del ajuste de ambas versiones en una misma muestra. Además, no se han estudiado estos instrumentos en la población Argentina. Para cumplimentar con los objetivos propuestos, se llevarán a cabo análisis factoriales exploratorios y confirmatorios de ambas escalas, lo que permitirá apreciar cuál de los dos inventarios ajusta mejor a los datos locales. Dado que el léxico Portugués es más cercano al de Argentina, se hipotetiza que la versión reducida Portuguesa tendrá un mejor ajuste en comparación a la versión Inglesa.

\section{Metodología}

\section{Participantes}

La muestra estuvo constituida por un total de 624 estudiantes universitarios. La selección de los participantes se realizó mediante un muestreo no probabilístico autoelegido. Este tipo de selección implica que la decisión de participar recae en la voluntad de los propios participantes (Bologna, 2012). Tanto la invitación a participar del estudio como la encuesta fueron enviadas vía web. La muestra quedo conformada por estudiantes pertenecientes a distintas unidades académica tanto privadas como públicas (e.g. Psicología; Abogacía; Medicina; Artes; Ingeniería; Letras). Del total de la muestra, el 74,4\% eran mujeres, mientras que el 25,6\% restante eran varones. Por último, las edades de los participantes estuvieron comprendidas entre los 17 y los 55 años $(M=25.25 ; D E=7.07)$.

\section{Instrumentos}

Se aplicó a los participantes la adaptación realizada del PANAS en Córdoba, Argentina (Flores Kanter \& Medrano, 2016; Medrano, et al., 2015). Siguiendo la escala original propuesta por Watson, Clark y Tellegen (1988), la misma consiste en 20 ítems, 10 que miden estados afectivos positivos y 10 que miden estados afectivos negativos. En el estudio exploratorio de la escala (Medrano, et al. 2015) se observó la existencia de dos factores que explican en conjunto un $36 \%$ de la variabilidad del instrumento, obteniéndose índices de consistencia interna aceptables $(\alpha=.84$ para Afecto Negativo; $\alpha=.75$ para Afecto Positivo). Los estudios confirmatorios posteriores (Flores Kanter \& Medrano, 2016) dieron sustento a esta estructura de dos factores relativamente independientes. En el presente estudio las instrucciones dadas a los participantes referían tanto al estado afectivo (i.e. la intensidad afectiva vivenciada en este preciso 
momento) como al rasgo afectivo (i.e. intensidad afectiva experimentada en el último mes). Asimismo, se ha evidenciado validez concurrente de esta escala al encontrar relaciones significativas con el engagement (Medrano, Moretti, \& Ortiz, 2015).

\section{Procedimiento}

Los participantes del estudio fueron invitados a participar vía web, en donde se les informó acerca de los objetivos de la investigación y la participación voluntaria a la misma. Asimismo, se les envió la escala PANAS vía web para ser respondida.

Luego de obtener las respuestas, los datos fueron cargados y analizados mediante los softwares estadísticos IBM SPSS 20, FACTOR (Lorenzo-Seva \& Ferrando, 2014), y AMOS 20. En primer lugar se procedió a subdividir la escala PANAS en dos muestras aleatorias de casos, que correspondieran al $50 \%$ aproximadamente del total de la muestra. Una submuestra se constituyo por un total de 306 participantes, y fue utilizada en el análisis factorial exploratorio (submuestra exploratoria). La segunda submuestra, compuesta por un total de 318 participantes, se utilizó a los fines del análisis factorial confirmatorio (submuestra confirmatoria). En segundo lugar, se seleccionaron, para cada submuestra, los ítems correspondientes a las versiones reducidas del PANAS. De esta forma, para verificar el ajuste de la versión Inglesa se seleccionaron de la escala total los siguientes ítems: Alerta, Inspirado, Determinado, Atento, Activo, Afligido, Hostil, Avergonzado, Nervioso, y Temeroso. Mientras que para verificar el ajuste de la versión Portuguesa, se seleccionaron de la escala total los siguientes ítems: Entusiasmado, Inspirado, Determinado, Activo, Interesado, Asustado, Temeroso, Afligido, Nervioso y Culpable.

Para llevar a cabo el análisis factorial exploratorio se tuvieron en cuenta las consideraciones brindadas por Ferrando y Lorenzo-Seva (2014) y Lloret-Segura, FerreresTraver, Hernández-Baeza y Tomás-Marco (2014). En función de los aportes de estos autores se considerará, a) la matriz de correlaciones producto-momento de Pearson, dado que se obtuvieron índices de asimetría y curtosis en su mayoría menores que 1 ; b) el análisis paralelo, uno de los criterios objetivos más recomendados para la determinación del número de factores; c) el método de estimación de mínimos cuadrados no ponderados (i.e. Unweighted Least Square; ULS); y d) rotación oblicua Promín que contempla la posibilidad de que los factores a estimar correlacionen entre sí. Por último, para verificar cuál escala presenta mejor ajuste se considerará la comunalidad de los ítems (Hair, Anderson, Tatham, \& Black, 1999); la proporción de varianza explicada por los factores obtenidos (Lorenzo-Seva, 2013); así como la simplicidad factorial lograda, verificada ésta última mediante la carga de los ítems a los factores luego de la rotación (i.e. presencia de ítems con cargas mayores a .30 en más de un factor) y el índice de simplicidad de carga (Lorenzo-Seva, 2003).

Para el análisis factorial confirmatorio $(A F C)$ se calcularon varios índices de ajustes globales para verificar la adecuación de los modelos. Concretamente, las estimaciones se llevaron a cabo mediante el método de máxima verosimilitud (i.e. Maximum Likelihood; $M L$ ), siendo este método confiable siempre y cuando la curtosis multivariada calculada por AMOS no supere el valor de 70 (Ayán \& Díaz, 2008); y se calcularon medidas de ajustes absolutos (i.e. chi cuadrado y RMSEA) y medidas de ajustes incrementales (i.e. $T L I$ y $C F I$ ). Los índices de ajustes mencionados son los más recomendados para su uso ( $\mathrm{Hu} \&$ Bentler, 1999), dada la confiabilidad que ofrecen en la estimación de los ajustes del modelo. Asimismo, se seguirán las recomendaciones de la literatura con respecto al valor de corte de los índices de ajustes para poder ser considerados satisfactorios (Hu \& Bentler, 1999); esto es, valores entre .05 y .08 para el índice RMSEA; y valores superiores a .90 para los índices $T L I$ y $C F I$.

\section{Resultados}

\section{Análisis Factorial Exploratorio}

En primer lugar se verificó la adecuación de la matriz de correlación para efectuar el análisis factorial exploratorio. Los resultados se describen en la Tabla 1. Puede apreciarse la mejor adecuación de la versión Portuguesa sobre la Inglesa, aunque ésta última alcance de igual modo valores aceptables. 
Tabla 1. Adecuación de las matrices. Test KMO y Estadístico de Bartlett.

\begin{tabular}{lcc}
\hline $\begin{array}{l}\text { Versión de } \\
\text { Escala }\end{array}$ & KMO & $\begin{array}{c}\text { Estadístico de } \\
\text { Bartlett }\end{array}$ \\
\hline $\begin{array}{l}\text { Portuguesa } \\
\text { Estado }\end{array}$ & .80 & $1214.3^{*}$ \\
$\begin{array}{l}\text { Portuguesa } \\
\text { Rasgo }\end{array}$ & .83 & $1284.1^{*}$ \\
Inglesa Estado & .77 & $865.8^{*}$ \\
Inglesa Rasgo & .77 & $914.6^{*}$ \\
\hline Nota $* p<.001, n=306$, & &
\end{tabular}

Se procederá ahora a llevar a cabo el análisis factorial exploratorio, mediante el método de estimación ULS. Para todas las versiones de las escalas, el análisis paralelo dio cuenta de dos factores que explican al conjunto de los ítems. En las siguientes tablas se sintetizan los resultados encontrados para la versión Portuguesa (Tabla 2 y 3) e Inglesa (Tabla 4 y 5). Para la saturaciones de los ítems en cada uno de los factores se tuvo en cuenta la matriz rotada de configuración, mientras que para la comunalidad presente en los ítems se tomará la carga de la matriz no rotada.

Tabla 2. Saturaciones factoriales, comunalidad, consistencia interna, varianza explicada, índice de simplicidad de carga, y correlaciones entre los factores. Versión Portuguesa. Estado Afectivo.

\begin{tabular}{|c|c|c|c|}
\hline \multirow[b]{2}{*}{ Ítems } & \multicolumn{2}{|c|}{ Saturación } & \multirow[b]{2}{*}{ Comunalidad } \\
\hline & $\begin{array}{l}\text { Afecto } \\
\text { Positivo }\end{array}$ & $\begin{array}{c}\text { Afecto } \\
\text { Negativo }\end{array}$ & \\
\hline Interesado & .569 & .033 & .326 \\
\hline Afligido & -.044 & .634 & .403 \\
\hline Culpable & -.114 & .660 & .445 \\
\hline Asustado & -.006 & .822 & .676 \\
\hline Entusiasmado & .798 & .048 & .641 \\
\hline Inspirado & .672 & .037 & .454 \\
\hline Nervioso & .153 & .700 & .520 \\
\hline Decidido & .777 & -.058 & .604 \\
\hline Activo & -.026 & 692 & .478 \\
\hline Temeroso & .829 & -.032 & .686 \\
\hline $\begin{array}{l}\text { Alfa de } \\
\text { Cronbach }\end{array}$ & .85 & .83 & \\
\hline $\begin{array}{l}\text { Varianza } \\
\text { Explicada }\end{array}$ & .61 & & \\
\hline LS & .82 & & \\
\hline Correlación I-F & .03 & & \\
\hline $\begin{array}{l}\text { Nota. } n=306 . \quad \\
\text { F=Inter-factor. } \\
\text { diferenciar las } \\
\text { respectivos facto }\end{array}$ & $\begin{array}{l}\text { Indice c } \\
\text { formato } \\
\text { gas fac } \\
\text { extraído }\end{array}$ & $\begin{array}{l}\text { simplicida } \\
\text { negrita } \\
\text { ales más }\end{array}$ & $\begin{array}{l}\text { de carga; I- } \\
\text { aplicó para } \\
\text { altas en los }\end{array}$ \\
\hline
\end{tabular}

Tabla 3. Saturaciones factoriales, comunalidad, consistencia interna, varianza explicada, índice de simplicidad de carga, y correlaciones entre los factores. Versión Portuguesa. Rasgo Afectivo.

\begin{tabular}{|c|c|c|c|}
\hline \multirow[b]{2}{*}{ Ítems } & \multicolumn{2}{|c|}{ Saturación } & \multirow[b]{2}{*}{ Comunalidad } \\
\hline & $\begin{array}{c}\text { Afecto } \\
\text { Positivo }\end{array}$ & $\begin{array}{c}\text { Afecto } \\
\text { Negativo }\end{array}$ & \\
\hline Interesado & .723 & .051 & .511 \\
\hline Afligido & -.022 & .592 & .357 \\
\hline Culpable & -.082 & .610 & .399 \\
\hline Asustado & -.025 & .803 & .654 \\
\hline Entusiasmado & .825 & .020 & .674 \\
\hline Inspirado & .711 & -.037 & .517 \\
\hline Nervioso & .085 & .696 & .468 \\
\hline Decidido & .756 & -.036 & .583 \\
\hline Activo & .724 & .050 & .512 \\
\hline Temeroso & .012 & .850 & .718 \\
\hline $\begin{array}{l}\text { Alfa de } \\
\text { Cronbach }\end{array}$ & .86 & .84 & \\
\hline $\begin{array}{l}\text { Varianza } \\
\text { Explicada }\end{array}$ & .63 & & \\
\hline LS & .88 & & \\
\hline Correlación I-F & -.19 & & \\
\hline $\begin{array}{l}\text { Nota. } n=306 . \\
\mathrm{F}=\text { Inter-factor. } \\
\text { diferenciar las } \\
\text { respectivos facto }\end{array}$ & $\begin{array}{l}\text { Indice d } \\
\text { formato } \\
\text { gas fac } \\
\text { extraído }\end{array}$ & $\begin{array}{l}\text { implicida } \\
\text { negrita } \\
\text { ales más }\end{array}$ & $\begin{array}{l}\text { de carga; I- } \\
\text { aplicó para } \\
\text { altas en los }\end{array}$ \\
\hline
\end{tabular}

Tabla 4. Saturaciones factoriales, comunalidad, consistencia interna, varianza explicada, índice de simplicidad de carga, y correlaciones entre los factores. Versión Inglesa. Estado Afectivo.

\begin{tabular}{|c|c|c|c|}
\hline \multirow[b]{2}{*}{ Ítems } & \multicolumn{2}{|c|}{ Saturación } & \multirow[b]{2}{*}{ Comunalidad } \\
\hline & $\begin{array}{c}\text { Afecto } \\
\text { Positivo }\end{array}$ & $\begin{array}{c}\text { Afecto } \\
\text { Negativo }\end{array}$ & \\
\hline Afligido & -.116 & .651 & .426 \\
\hline Hostil & -.056 & .448 & .200 \\
\hline Alerta & .355 & .323 & .248 \\
\hline Avergonzado & -.145 & .583 & .348 \\
\hline Inspirado & .583 & .071 & .351 \\
\hline Nervioso & .098 & .749 & .582 \\
\hline Decidido & .829 & -.033 & .683 \\
\hline Atento & .695 & -.042 & .481 \\
\hline Activo & .726 & .021 & .530 \\
\hline Temeroso & -.108 & .729 & .530 \\
\hline $\begin{array}{l}\text { Alfa de } \\
\text { Cronbach }\end{array}$ & .77 & .76 & \\
\hline $\begin{array}{l}\text { Varianza } \\
\text { Explicada }\end{array}$ & .54 & & \\
\hline LS & .63 & & \\
\hline Correlación I-F & .08 & & \\
\hline $\begin{array}{l}\text { Nota. } n=306 . \\
\text { F=Inter-factor. } \\
\text { diferenciar las } \\
\text { respectivos facto }\end{array}$ & $\begin{array}{l}\text { Indice } d \\
\text { formato } \\
\text { rgas fac }\end{array}$ & $\begin{array}{l}\text { simplicida } \\
\text { negrita } \\
\text { iales más }\end{array}$ & $\begin{array}{l}\text { de carga; I- } \\
\text { aplicó para } \\
\text { altas en los }\end{array}$ \\
\hline
\end{tabular}


Tabla 5. Saturaciones factoriales, comunalidad, consistencia interna, varianza explicada, índice de simplicidad de carga, y correlaciones entre los factores. Versión Inglesa. Rasgo Afectivo.

\begin{tabular}{|c|c|c|c|}
\hline \multirow[b]{2}{*}{ Ítems } & \multicolumn{2}{|c|}{ Saturación } & \multirow[b]{2}{*}{ Comunalidad } \\
\hline & $\begin{array}{c}\text { Afecto } \\
\text { Positivo } \\
\end{array}$ & $\begin{array}{c}\text { Afecto } \\
\text { Negativo }\end{array}$ & \\
\hline Afligido & -.029 & .658 & .438 \\
\hline Hostil & -.003 & .450 & .203 \\
\hline Alerta & .482 & .345 & .306 \\
\hline Avergonzado & -.081 & .526 & .295 \\
\hline Inspirado & .620 & -.116 & .418 \\
\hline Nervioso & .083 & .666 & .435 \\
\hline Decidido & .768 & -.069 & .610 \\
\hline Atento & .770 & .149 & .583 \\
\hline Activo & .761 & .014 & .576 \\
\hline Temeroso & -.015 & .759 & .580 \\
\hline $\begin{array}{l}\text { Alfa de } \\
\text { Cronbach }\end{array}$ & .75 & .80 & \\
\hline $\begin{array}{l}\text { Varianza } \\
\text { Explicada }\end{array}$ & .55 & & \\
\hline $\mathrm{LS}$ & .69 & & \\
\hline Correlación I-F & -.14 & & \\
\hline
\end{tabular}

Considerando los valores comparativos propuestos para el análisis factorial exploratorio (Tabla 2-5), es posible observar que a) en la Versión Portuguesa se logró una mayor proporción de varianza explicada por los factores considerados, considerando el EA (.61) y el RA (.63), en comparación a la Versión Inglesa $(\mathrm{EA}=.54 ; \mathrm{RA}=.55) ; \mathrm{b})$ en la Versión Portuguesa se alcanzó un mayor índice de simplicidad de carga (LS), lo que da cuenta de una estructura factorial más simple, teniendo en cuenta el EA $(L S=.82)$ como el RA $(L S=.88)$, en comparación a la Versión Inglesa ( $L S \mathrm{EA}=.63 ; L S \mathrm{RA}=.69$ ); y c) los coeficientes alfas de Cronbach dan cuenta de una mejor consistencia interna en la Versión Portuguesa, respecto al EA ( $\alpha$ Afecto Positivo=.85; $\alpha$ Afecto Negativo=.83) y RA $(\alpha$ Afecto Positivo=.86; $\alpha$ Afecto Negativo=.84), en comparación a la Versión Inglesa, en relación al EA $(\alpha$ Afecto Positivo=.77; $\alpha$ Afecto Negativo=.76) como al RA $(\alpha$ Afecto Positivo=.75; $\alpha$ Afecto Negativo=.80).

\section{Análisis Factorial Confirmatorio}

Luego se procedió con la submuestra confirmatoria $(n=318)$ con el objetivo de llevar a cabo el análisis factorial confirmatorio. Para todos los casos, se verifico un buen ajuste del modelo bifactorial del afecto, es decir los ítems se agrupan en dos dimensiones representantes del Afecto Positivo y Negativo. Los resultados se detallan en la tabla siguiente (Tabla 6).

Como puede apreciarse en la Tabla 6, los índices de ajustes calculados mediante el análisis factorial confirmatorio también evidenciaron un mejor ajuste de la Versión Portuguesa, mostrando diferencias favorables en todos los índices calculados, en función del EA $\left(\Delta \chi^{2}=16.51\right.$; $\triangle T L I=.04 ; \triangle C F I=.03 ; \triangle R M S A=.01)$ como del RA $\left(\Delta \chi^{2}=40.68 ; \Delta T L I=.07 ; \Delta C F I=.05 ; \triangle R M S E A=.03\right)$. Finalmente, las correlaciones entre los factores latentes (i.e. Afecto Positivo y Afecto Negativo) pueden considerarse como muy bajas y nulas (valores entre .05 y $-.13, p>.05$ ) siguiendo los criterios de Bologna (2012).

\section{Discusión}

El presente estudio se propuso adaptar al contexto Argentino una Versión Reducida de la Escala PANAS (PANAS-VR). Para esto, se propuso comparar el ajuste de dos versiones distintas, una Versión Inglesa y otra Portuguesa.

Como se había hipotetizado, la Versión Portuguesa es la que presento un mejor ajuste al contexto local. Lo anterior quedó evidenciado en los sucesivos análisis factoriales exploratorios y confirmatorios. Este hecho puede explicarse en base a las similitudes lingüísticas y culturales (Muñiz, Elosua \& Hambleton, 2013). En efecto hay más similitud léxicas entre Argentina y la población Portuguesa que la que puede encontrarse con el idioma inglés.

Tomando ahora en consideración solamente la Versión Portuguesa, la misma presentó adecuada evidencia de validez de constructo en las muestras estudiadas, tanto en la submuestra exploratoria como en la submuestra confirmatoria, aportando también así evidencia de validez cruzada. Con respecto al análisis factorial exploratorio, los resultados muestran cargas adecuadas en los respectivos factores, tanto en las mediciones del EA (cargas factoriales entre .57 y .83 para el Afecto positivo, y entre .63 y .82 para el Afecto Negativo) como en las del RA (cargas factoriales entre .72 y .82 para el Afecto positivo, y entre .59 
Tabla 6. Indicadores de Ajustes en función de las distintas versiones del PANAS Reducido.

\begin{tabular}{lcccccc}
\hline Versiones & Curtosis M. & $\chi^{2}$ & $g l$ & $T L I$ & $C F I$ & RMSEA \\
\hline Portuguesa EA & 14.84 & $94.46^{*}$ & 34 & .94 & .95 & .07 \\
Portuguesa RA & 15.07 & $91.74^{*}$ & 34 & .95 & .96 & .07 \\
Inglesa EA & 14.59 & $110.97^{*}$ & 34 & .90 & .92 & .08 \\
Inglesa RA & 8.92 & $132.42^{*}$ & 34 & .88 & .91 & .10 \\
\hline Dif. Ver. EA & & 16.51 & & .04 & .03 & .01 \\
Dif. Ver. RA & & 40.68 & & .07 & .05 & .03
\end{tabular}

Nota. ${ }^{*} p<.001, n=318$; EA=Estado Afectivo; RA=Rasgo Afectivo; Dif. Ver. EA=Diferencias en el Ajuste de las Versiones Portuguesa e Inglesa, respecto al Estado Afectivo; Dif. Ver. RA=Diferencias en el Ajuste de las Versiones Portuguesa e Inglesa, respecto al Rasgo Afectivo.

y .85 para el Afecto Negativo). Sumado a lo anterior, los coeficientes alfa de Cronbach dan cuenta de una consistencia interna adecuada en los respectivos factores, en las mediciones del EA ( $\alpha$ Afecto Positivo $=.85 ; \alpha$ Afecto Negativo=.83) y RA $(\alpha$ Afecto Positivo=.86; $\alpha$ Afecto Negativo=.84). Por su parte, tal como puede apreciarse en el análisis factorial confirmatorio la Versión Portuguesa alcanza adecuados índices de ajuste, en relación al EA (TLI=.94; $C F I=.95$; RMSEA=.07) como al RA (TLI=.95; CFI=.96; RMSEA=.07).

De este modo, se ha verificado el ajuste del modelo originalmente propuesto por Watson (2000) de dos factores relativamente independientes (i.e. Afecto Positivo y Negativo). En términos teóricos, los resultados apoyan la existencia de dos factores claramente diferenciados (Watson, 2000; Gray \& Watson, 2007). Siguiendo las evidencias de las neurociencias, si bien se han postulados estructuras y mecanismos cerebrales propios de cada estado afectivo, como ser la ansiedad y la depresión (Davidson, 2000; Keller et al. 2000), neuroanatómicamente se han identificado también ciertas estructuras cerebrales más ligadas a estos factores comunes de Afectividad o Valencia Negativa y de Afectividad o Valencia Positiva (Berridge, 2003; Davidson, Pizzagalli, Nitschke, \& Kalin, 2003; Davidson, 2000; Heller, 1993; Suls, 2017). En el caso del Afecto Negativo, una de estas estructuras es la amígdala, la cual ha demostrado activarse preferentemente al experimentar afectos negativos. Lo anterior no implica necesariamente que sea una estructura netamente involucrada a los afectos negativos, pero sí que tiene una funcionalidad preferentemente relacionada a estos afectos
(Kristen, Satpute, Wager, Weber, \& Barrett, 2016). Para el caso del Afecto Positivo la estructura involucrada es el núcleo accumbens, identificado como el sistema de recompensa y placer (Berridge, 2003).

La versión reducida propuesta por Galinha, et al., (2014) puede considerarse equivalente en términos de propiedades psicométricas a las versiones completas ya adaptadas en el contexto argentino por Medrano, et al. (2015) y Flores Kanter \& Medrano (2016). La ventaja que presenta esta versión reducida es que facilita las respuestas en cuestionarios extensos que envuelven muchas variables, o en contextos aplicados donde hay limitaciones de tiempo, como puede ser el organizacional o educacional por ejemplo (Galinha, et al. 2014; Moreira \& Gamboa, 2016).

En suma, los resultados descritos avalan la utilización en el contexto Argentino de la Versión Reducida del PANAS (PANAS-VR-ARG), integrada por los ítems de la Versión Portuguesa (Galinha, et al., 2014). La misma puede ser aplicada tanto en estudios que deseen indagar el Estado Afectivo (i.e. intensidad afectiva en este preciso momento) como el Rasgo Afectivo (i.e. intensidad afectiva en el último mes). Finalmente, sería conveniente replicar los análisis en otras muestras no pertenecientes a estudiantes universitarios, así como también llevar a cabo análisis de validez convergente-discriminante, y de test-criterio. Por otra parte, sería relevante que futuras investigaciones considerasen poner a prueba modelos alternativos al modelo bifactorial, tal como se ha llevado a cabo recientemente en Argentina para la versión completa del PANAS (Flores Kanter \& Medrano, 2016). Además, es importante considerar que en la presente 
investigación se tomó la versión completa del PANAS a partir de lo cual se analizó la adecuación de las versiones cortas. Futuras investigaciones deberían verificar las propiedades psicométricas de estas versiones considerando directamente el formato breve de las escalas, dado que el contenido del instrumento (e.g. ítems presentados, formulación de consignas) puede dar lugar a respuestas distintas de los participantes (Tornimbeni, Pérez, \& Olaz, 2008).

\section{Referencias}

Ayán, M. N. R., \& Díaz, M. A. R. (2008). Atenuación de la asimetría y de la curtosis de las puntuaciones observadas mediante transformaciones de variables: Incidencia sobre la estructura factorial. Psicológica, 29, 205-227.

Berridge, K. C. (2003). Pleasures of the brain. Brain and Cognition, 52(1), 106-128. doi:https://doi.org/10.1016/S0278-2626(03)00014-9

Bologna, E. (2012). Estadística para psicología y educación. Cordoba: Editorial Brujas.

Bower, G. H. \& Forgas, J. P. (2001). Mood and social memory. En Forgas, J. Handbook of Affect and Social Cognition (pp. 95-120). New York: Psychology Press.

Damasio, A. R. (2005). En busca de Spinoza. Neurobiología de la emoción y los sentimientos. Barcelona: Crítica.

Davidson, R. J. (2000). Affective style, mood, and anxiety disorders: An affective neuroscience approach. En R. J. Davidson, Anxiety, Depression, and Emotion. New York: Oxford University Press.

Davidson, R. J., Pizzagalli, D., Nitschke, J. B. \& Kalin, N. H. (2003). Parsing the subcomponents of emotion and disorders of emotion: Perspectives from affective neuroscience. En Davidson, R. J.; Scherer, K. R \& Goldsmith, H. H. Handbook of affective sciences (pp. 8-25). New York: Oxford University Press.

Davidson, R. J.; Scherer, K. R \& Goldsmith, H. H. (2003). Handbook of affective sciences. New York: Oxford University Press.

Eich, E., Kihlstrom, J., Bower, G., Forgas, J., \& Niedenthal, P. (2003). Cognición y Emoción. España: Descleé De Brouwer
Ferrando, P. J., \& Lorenzo-Seva, U. (2014). El análisis factorial exploratorio de los ítems: Algunas consideraciones adicionales. Anales de psicología, 30 (3), 1170-1175.

Flores Kanter, P. E. \& Medrano, L. (2016). El afecto y sus dimensiones: Contrastes de modelos ortogonales y oblicuos mediante análisis factorial confirmatorio de la escala PANAS. LIBERABIT, 22(2), 173-184.

Flores Kanter, P. E., Medrano, L., \& Conn, H. (2015). Is it possible that mood affects selfconception? Analysis through an approach based on natural semantic networks. International Journal of Behavioral Research \& Psychology (IJBRP), 3 (5), 114-120.

Flores Kanter, P. E., Medrano, L. \& Manoiloff, L. (2014). Estados de ánimo y juicios de autoconcepto en universitarios: Análisis desde un abordaje basado en Redes Semánticas Naturales. Interamerican Journal of Psychology (IJP), 48 (3), 291-296.

Forgas, J. P. (2001). Handbook of affect and social cognition. New York: Psychology Press.

Forgas, J. P. (2003). Affective influences on attitudes and judgments. En Davidson, R. J.; Scherer, K. R \& Goldsmith, H. H. Handbook of affective sciences (pp. 596-619). New York: Oxford University Press.

Galinha, I. C., Pereira, C. R., \& Esteves, F. G. (2013). Confirmatory factor analysis and temporal invariance of the positive and negative affect schedule (PANAS). Psicologia: Reflexão e Crítica, 26 (4), 671-679.

Galinha, I. C., Pereira, C. R., \& Esteves, F. G. (2014). Versao reduzida da escala portuguesa de afeto positivo e negativo - PANAS-VRP: Analise fatorial confirmatoria e invariancia temporal. Revista PSICOLOGIA, 28 (1), 53-65.

Gargurevich, R. (2010). Propiedades psicométricas de la versión internacional de la Escala de Afecto Positivo y Negativo-forma corta (I- Spanas SF) en estudiantes universitarios. Persona, 13, 31-42.

Gray, E. K., \& Watson, D. (2007). Assessing positive and negative affect via self-report. En Coan, J. A. \& Allen, J. J. B. Handbook of emotion elicitation and assessment (pp. 171184). New York: Oxford University Press.

Gyollai, A., Simor, P., Köteles, F., \& Demetrovics, Z. (2011). Psychometric 
properties of the Hungarian version of the original and the short form of the Positive and Negative Affect Schedule (PANAS). Neuropsychopharmacologia Hungarica, 13 (2), 73-79.

Hair, J. F., Anderson, R. E., Tatham, R. L., \& Black, W. C. (1999). Análisis Multivariante. Madrid: Person Prentice Hall.

Heller, W. (1993). Neuropsychological mechanisms of individual differences in emotion, personality, and arousal. Neuropsychology, 7(4), 47-189.

Hervás, G. \& Vázquez, C. (2006). La regulación afectiva: Modelos, investigación e implicaciones para la salud mental y física. Revista de psicología general y aplicada 59(1-2), 9-36.

Hu, L., \& Bentler, P. M. (1999). Cutoff criteria for fit indexes in covariance structure analysis: Conventional criteria versus new alternatives. Structural Equation Modeling: A Multidisciplinary Journal, 6 (1), 1-55.

Karima, J., Weisz, R., Rehman, S. U. (2011). International positive and negative affect schedule short-form (I-PANAS-SF): Testing for factorial invariance across cultures. Procedia Social and Behavioral Sciences, 15, 2016-2022.

Keller, J., Bhargava, T., Gergen, J. A., Miller, G. A., Nitschke, J. B., Deldin, P. J. \& Heller, W. (2000). Neuropsychological differentiation of depression and anxiety. Journal of Abnormal Psychology, 109(1), 3-10. doi: 10,1037//0021-S43X. 109.1.3

Kristen, A. L., Satpute, A. B., Wager, T. D., Weber, J. \& Barrett, L. F. (2016). The brain basis of positive and negative affect: Evidence from a meta-analysis of the human neuroimaging literature. Cerebral Cortex, 26, 1910-1922. doi: 10.1093/cercor/bhv001

Loewenstein, G., \& Lerner, J. S. (2003). The role of affect in decision making. En Davidson, R. J.; Scherer, K. R \& Goldsmith, H. H. Handbook of affective sciences (pp. 619-643). New York: Oxford University Press.

Lorenzo-Seva, U. (2003). A factor simplicity index. PSYCHOMETRIKA, 68 (1), 49-60.

Lloret-Segura, S., Ferreres-Traver, A., HernándezBaeza, A. \& Tomás-Marco, I. (2014). El análisis factorial exploratorio de los ítems:
Una guía práctica, revisada y actualizada. Anales de psicología, 30 (3), 1151-1169.

MacGaught, J. L., \& Cahill, L. (2009). Emotion and memory: Central and peripheral contributions. En Davidson, R. J.; Scherer, K. R \& Goldsmith, H. H. Handbook of affective sciences (pp. 93-117). New York: Oxford University Press.

Medrano, L., Flores Kanter, P. E., Pereno, G., \& Moretti, L. (2015). Effects of induction of positive and negative emotional states in the beliefs of academic self-efficacy in college students. Psicología Educativa, 22(2), 135141. doi:10.1016/j.pse.2015.03.003.

Medrano, L. A., Flores Kanter, P. E., Trógolo, M., Curarello, A., \& Gonzales, J. (2015). Adaptación de la Escala de Afecto Positivo y Negativo (PANAS) para la población de Estudiantes Universitarios de Córdoba. Anuario de Investigaciones de la Facultad de Psicología, 2(1), 22-36.

Medrano, L. A., Moretti, L. S., \& Ortiz, A. (2015). Medición del engagement académico en estudiantes universitarios. Revista Iberoamericana de Diagnóstico y Evaluación - e Avaliação Psicológica, 2(40), 114-123.

Merz, E. L., Malcarne, V. L, Roesch, S. C., Ko, C. M., Emerson, M., Roma, V. G., \& Sadler, G. R. (2013). Psychometric properties of Positive and Negative Affect Schedule (PANAS) original and short forms in an African American community simple. Journal of Affective Disorders, 151, 942-949.

Moreira, J. M. \& Gamboa, P. (2016). Inventário de estados afetivos-reduzido: Uma medida multidimensional breve de indicadores emocionais de ajustamento. Revista Iberoamericana de Diagnóstico y Evaluación - e Avaliação Psicológica, 41(1), 132-144.

Moriondo, M., De Palma, P., Medrano, L. A., \& Murillo, P. (2012). Adaptación de la Escala de Afectividad Positiva y Negativa (PANAS) a la población de adultos de la ciudad de Córdoba: análisis psicométricos preliminares. Universitas Psychologica, 11 (1), 187-196.

Muñiz, J., Elosua, P., \& Hambleton, R. K. (2013). Directrices para la traducción y adaptación de los tests: Segunda edición. Psicothema, 25(2), 151-157. doi:10.7334/psicothema2013.24 
Reisberg, D., \& Hertel, P. (2004). Memory and emotion. New York: Oxford University Press.

Rolls, E. T. (2014). Emotion and decision-making explained: Précis. Cortex.

Suls, J. (2017). Toxic affect: Are anger, anxiety, and depression independent risk factors for cardiovascular disease? Emotion Review, 112. doi: $10.1177 / 1754073917692863$

Terracciano, A., Mc Crae, R. R., \& Costa, P. T. (2003). Factorial and construct validity of the Italian positive and negative affect schedule (PANAS). European Journal of Psychological Assessment, 19 (2), 131-141.

Thompson, E. R. (2007). Development and validation of an Internationally Reliable Short-Form of the Positive and Negative Affect Schedule (PANAS). Journal of CrossCultural Psychology, 38 (2), 227-242.

Tornimbeni, S., Pérez, E. \& Olaz, F. (2008). Introducción a la Psicometría. Buenos Aires, Argentina: Paidós.

Tuccitto, D. E., Giacobbi, P. R., \& Leite, W. L. (2010). The Internal Structure of Positive and Negative Affect: A confirmatory factor analysis of the PANAS. Educational and Psychological Measurement, 70 (1), 125-141.

Watson, D. (2000). Mood and temperament. New York: The Guilford Press.

Watson, D., Clark, L. A., \& Tellegen, A. (1988). Development and validation of brief measures of positive and negative affect: The PANAS scales. Journal of Personality and Social Psychology, 54, 1063-1070. 